Journal of

Dentistry and Oral Health

\title{
Influence of Tooth Brush Grips and Brushing Techniques on Plaque Removal Efficacy
}

\section{Khalid Jamal Alanazi ${ }^{1, *}$, Syed Abdul Subhan ${ }^{2}$, Hassan M Alshehri ${ }^{3}$, Mousa M Aljaload ${ }^{4}$, Mohammad H} Aljafary $^{5}$, Mohammed Muraybid Alazmi ${ }^{5}$, Albaker A Fawaz ${ }^{4}$

${ }^{1}$ Post Graduate Resident, Department of Pediatric Dentistry, Riyadh Colleges of Dentistry and Pharmacy, Riyadh, Kingdom of Saudi Arabia

${ }^{2}$ Lecturer, Department of Preventive Dentistry, Riyadh Colleges of Dentistry and Pharmacy, Riyadh, Kingdom of Saudi Arabia

${ }^{3}$ Post Graduate Resident, Department of orthodontics, Riyadh Colleges of Dentistry and Pharmacy, Riyadh, Kingdom of Saudi Arabia

${ }^{4}$ General Practitioner, Ministry of Health, Kingdom of Saudi Arabia

${ }^{5}$ Post Graduate Resident, Department of Restorative Dentistry, Riyadh Colleges of Dentistry and Pharmacy

${ }^{\star}$ Corresponding author: Khalid Jamal Alanazi, Riyadh Colleges of Dentistry and Pharmacy 8502 Al Olaya, Riyadh 12611 Kingdom of Saudi Arabia; Tel: +966507977704; E-mail: k.j.h.o@windowslive.com

Received Date: February 16, 2017; Accepted Date: March 3, 2017; Published Date: March 5, 2017

Citation: Khalid Jamal Alanazi, et al. (2017) Influence of Tooth Brush Grips and Brushing Techniques on Plaque Removal Efficacy. J Dent Oral Health 4: 1-7.

\begin{abstract}
Introduction: Tooth brushing is an essential element of an effective plaque control program, and attaining optimal oral health would depend on the efficacy of the method used and the ease of carrying out the procedure.

Aim: To explore the effects of distal oblique, oblique and precision toothbrush grips, and two methods of brushing, the modified Stillman's technique and the modified Bass technique, on plaque removal.

Methodology: Forty male subjects aged between 18 and 22 years participated in the study. Data was obtained using video recordings of subjects as they conducted their normal tooth brushing sessions. We observed the manner in which they gripped their toothbrushes, and the brushing techniques used. This information was analyzed to show any associations between the type of grip used and the efficacy of plaque removal, using a quantitative analysis of plaque scores which were recorded at periodic intervals. In addition, the study investigated the changes in plaque scores when the subjects used either the modified Stillman's brushing technique or the modified Bass technique. The experimental design was double-blind.

Result: There was a significant difference in plaque scores when different toothbrush grips were used, and a significant difference in plaque scores was also observed between the two brushing techniques investigated.

Conclusion: This study confirmed that both an oblique grip and a distal oblique grip are efficacious for the removal of plaque. The modified Bass technique in combination with either an oblique distal grip or an oblique grip was significantly superior to the modified Stillman's technique with respect to plaque removal.
\end{abstract}

Keywords: Bass technique; Distal oblique grip; Oblique grip; Plaque; Stillman's technique

C2017 The Authors. Published by the JScholar under the terms of the Creative Commons Attribution License http://creativecommons.org/licenses/ by/3.0/, which permits unrestricted use, provided the original author and source are credited. 


\section{Introduction}

The negative correlation between poor tooth brushing, dental plaque and plaque-induced gingivitis, and dental caries has been a subject of much research. All dental professionals aim to help members of the public to attain optimal oral health, and one way of doing this is to emphasize the need to take precautionary measures [1]. A critical component of any preventive intervention for dental health, whether it targets groups or individuals, is a properly designed plaque control program. Any efforts to help the public to maintain oral health must include advice regarding the need to maintain oral cleanliness, because this helps to eliminate microbial plaque, thus inhibiting it from building up in the gingivae and teeth [2]. Tooth brushing is an essential element of an effective plaque control program, and we hypothesized that attaining optimal oral health would depend on the efficacy of the method used and the ease of carrying out the procedure. It is well established that, in children, tooth brushing is still the most efficient way of removing plaque in the long term [3] and normal tooth brushing is sufficient to prevent bacterial plaque [4]. However, when we buy a toothbrush, we rarely receive special instructions on how to use it, except where the manufacturer might include some instruction in the packaging.

Numerous studies investigating preventive dental strategies, such as those of Kimmelman et al. [5] and more recently Mathur et al. [6], have paid great attention to toothbrush designs and their ability to meet the needs of children. Other researchers have focused on the duration of tooth brushing. For instance, Salzer et al. [2] suggested that tooth brushing once per day is an adequate measure to prevent the development of inter-dental caries and gingivitis. Both the use of the correct toothbrush and compliance with the method advised are major determinants of the effectiveness of tooth brushing [3]. However, despite the increased research focus on methods of tooth brushing and its duration, little attention has been paid to the different ways in which people hold their toothbrushes when brushing their teeth.

Beals et al. [7] made an effort to document the ways in which people grip their toothbrushes during normal tooth brushing sessions. Their study outlined five main toothbrush grips, namely the power grip, distal oblique grip, precision grip, oblique grip, and spoon grip (Figure 1). The power grip and the distal oblique grip use the palm of the hand, while the remaining three grips rely on the fingers to grip the toothbrush. While a few studies have explored associations between the ways in which children grip their toothbrushes and their dexterity [8], less attention has been paid to associations between grip type and the effectiveness of plaque removal in adults.

A number of methods of tooth brushing can be used to remove dental plaque from the surface of the teeth; however, these methods differ in terms of their effectiveness and the time which is needed to master them. Due to their complexity, it is also possible that their effectiveness varies with time, according to the ability of the user to adhere to the instructions given. A few researchers have investigated the effectiveness of using different tooth brushing techniques on plaque removal, for example, a study by Arai et al,[9] compared the effectiveness of six methods. To generate evidence to support any of the existing tooth brushing methods, there is a need for a study comparing the efficacy with respect to plaque removal of different methods performed using different tooth brushing grips.

Although video recording techniques are popular for collecting data in developmental psychology, they are rarely used for research in the field of dentistry [10]. However, the advantage of video recording as a method of data collection is that it documents nonverbal behavior as well as generating a record of what the interviewee says. Therefore, given the nature of this current study, video recording was deemed to be an appropriate method for collecting data, enabling us to observe how the subjects gripped their toothbrushes, as well as any changes in grip over time, and the methods of brushing utilized. The current study was an observational exploration of the ways in which people grip their toothbrushes during their normal tooth brushing sessions. It recorded the time taken to brush the teeth and the technique used by adults in such sessions. It also explored relationships between the grip type and the effectiveness of plaque removal, and evaluated correlations over time between plaque reduction, brushing techniques and the duration of brushing for each subject.

So the aim of the present study was to explore the influences of different toothbrush grips on plaque removal, the modified Stillman's and the modified Bass tooth brushing techniques on plaque removal and the combination of tooth brushing technique and toothbrush grip on plaque removal.

\section{Materials and Methods}

The procedures followed in the study were in accordance with the ethical standards as described by Helsinki Declaration of 1975 that was revised in 2000. A total of 40 male students aged between 18 and 22 years volunteered to participate in this study. Each subject provided written informed consent. Subjects were required to meet certain standards to qualify for participation in this study, and those who failed to meet them were excluded.

The following exclusion criteria were applied: Students who:

a. Had an existing systemic disease that could affect their brushing

b. Had been prescribed non-steroidal anti-inflammatory drugs or antibiotics in the month prior to the study.

c. Were currently using a prosthetic appliance or were under orthodontic treatment.

d. Did not use a toothbrush for cleaning their teeth.

e. Had multiple dental caries or malocclusions, for example, due to tooth crowding.

f. Were already suffering from periodontal and gingival disease.

\section{Methodology}

This was a double-blind study with data collected at each of five visits conducted at a seven day intervals. The research team was blinded to the subjects' tooth brushing techniques and brushing grips, and received no information regarding how the division of subjects was undertaken to avoid researcher bias when recording the plaque index. 
Figure 1: Different types of tooth brush grip described by Beals et al

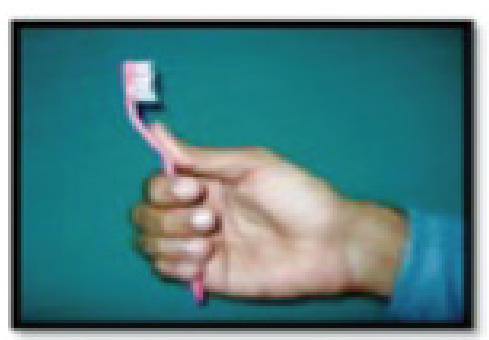

Distal oblique

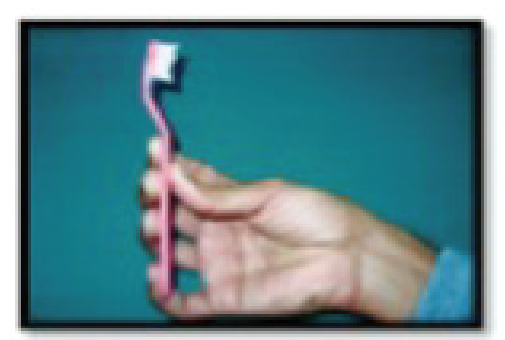

oblique

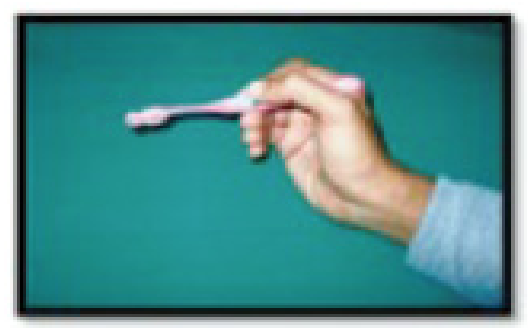

Spoon

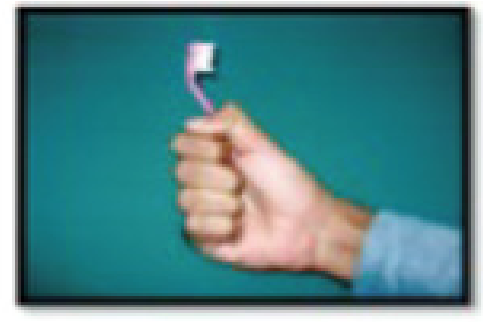

Power

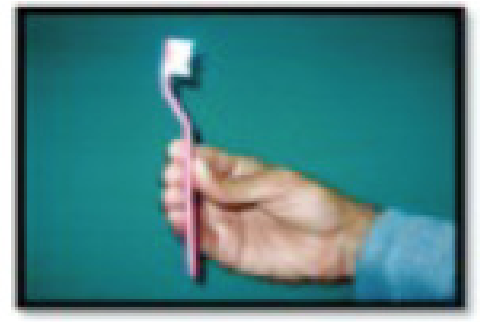

Precision
Visit 1:

The Quigley and Hein Plaque Index (TQHPI) were used, which is a modified protocol described by Turesky et al [11]. The subject rinses their mouth for one minute with a plaque disclosing solution (Colgate Disclotabs, Colgate -Palmolive, KSA) designed to reveal plaque, and then the first plaque score (Ps1) is recorded. The subjects were instructed to spread the toothpaste and clean their teeth as they normally would at home, and a camera placed ten feet from where each subject was brushing their teeth was used to record a video of the process. The same procedure used to calculate Ps1 was used again to calculate the plaque index after the first session of brushing (Ps2). The video records of the tooth brushing sessions were subsequently employed in analyzing the method used to grip the toothbrush, in accordance with the model described by Beals et al. [7], as well as the tooth brushing technique that each subject used. This process was then repeated after a period of seven days.

Visit 2:

The same procedure performed during the first visit was used, and the resulting plaque index scores were recorded as Ps3 and Ps4, respectively. At this second visit, the subjects were assigned to one of two groups, group A and group B. Group A was taught how to brush their teeth using the modified Stillman's technique, and group B was taught how to brush their teeth using the modified Bass technique.
Each subject was informed that they should use the designated technique for the next seven days, with the same toothpaste and toothbrush issued at the initial visit, for a brushing session lasting three minutes two times a day.

Visit 3:

Each subject was told to brush their teeth as they had been trained to during Visit 2, and video records of the tooth brushing session were obtained. Plaque scores (Ps5 and Ps6) were again recorded, using the same process utilized previously.

\section{Visit 4 and 5:}

At these visits, identical methodologies to visit 3 were used, and plaque scores were recorded as Ps7 to Ps10.

\section{Statistical Analysis}

The data derived from these experiments were analyzed using the SPSS software package (version 22.0, IBM Corp., Armonk, NY). The independent t-test was used to compare the differences in plaque scores associated with the variations in subjects' grips. To compare the plaque scores recorded at different intervals, the paired t-test was used. 


\section{Results}

The mean age of the 40 male subjects was $20.0 \pm 1.3$ years. Table 1 summarizes the different grips and brushing techniques used by the subjects. As indicated in the frequency column, the most common brushing technique used was horizontal scrubbing, and the most popular method of holding the brush was the distal oblique grip, followed by the oblique grip and then the precision grip. As shown in Table 2, each subject retained the same method of gripping the toothbrush after being instructed on how to use either the modified Bass technique or the modified Stillman's technique. Table 3 present the plaque index score comparisons for each visit, from the first to the fifth, both before brushing and after brushing, grouped according to the grip method used. There was a notable reduction in plaque scores before brushing, but it was not statistically significant; however, differences in plaque scores after the brushing session were statistically significant $(p<0.05)$ for all three types of grips.

Table 1: Frequency distribution of volunteers

\begin{tabular}{|c|c|c|}
\hline \multicolumn{3}{|c|}{ Grip } \\
\hline & Frequency & Percent \\
\hline Distal oblique & 22 & 55.0 \\
\hline Oblique & 11 & 27.5 \\
\hline Precision & 7 & 17.5 \\
\hline Total & 40 & 100.0 \\
\hline \multicolumn{3}{|c|}{ Brushing method visits 1 and 2} \\
\hline & Frequency & Percent \\
\hline Horizontal & 23 & 57.5 \\
\hline Vertical & 8 & 20.0 \\
\hline Circular & 9 & 22.5 \\
\hline Total & 40 & 100.0 \\
\hline
\end{tabular}

Table 2: Frequency distribution of subjects

\begin{tabular}{|c|c|c|}
\hline \multicolumn{3}{|c|}{ Grip } \\
\hline & Frequency & Percent \\
\hline Distal oblique & 22 & 55.0 \\
\hline Oblique & 11 & 27.5 \\
\hline Precision & 7 & 17.5 \\
\hline Total & 40 & 100.0 \\
\hline \multicolumn{3}{|c|}{ Brushing Technique visits 3,4 and 5} \\
\hline & Frequency & Percent \\
\hline Modified Bass & 20 & 50.0 \\
\hline Modified Stillman's & 20 & 50.0 \\
\hline Total & 40 & 100.0 \\
\hline
\end{tabular}

Table 4 are summaries of the comparisons of plaque scores before and after brushing during the third and fifth visits, according to the two different brushing techniques the subjects were taught at the second visit. There was a significant difference in plaque reduction when either the modified Stillman's technique or the modified Bass technique was used, both pre- and post-brushing. Table 5 show comparisons of plaque scores before brushing during both the third and fifth visits, according to the combination of brush grip and brushing technique used. A significantly greater reduction in the amount of plaque was observed when using the modified Bass technique with either an oblique grip or distal oblique grip as compared to using the modified Bass technique with the precision grip. While there were reductions in plaque scores when subjects used the modified Stillman's technique in conjunction with oblique, distal oblique, and precision grips, these differences were not statistically significant. 
Table 3:Comparison of the effects of three grips on plaque index

\begin{tabular}{|l|l|l|l|l|l|l|l|}
\hline & & PRE-1 & PRE-5 & sig. 2 TAILED & POST-1 & POST-5 & sig. 2 TAILED \\
\hline GRIP & $\begin{array}{l}\text { D I S T A L } \\
\text { OBLIQUE }\end{array}$ & $173.00 \pm 52.74$ & $159.30 \pm 48.64$ & NS & $168.50 \pm 45.00$ & $13.95 \pm 10.67$ & S \\
\hline & OBLIQUE & $180.00 \pm 49.37$ & $168.18 \pm 19.11$ & NS & $93.64 \pm 25.30$ & $16.45 \pm 9.68$ & S \\
\hline & PRECISION & $154.00 \pm 33.50$ & $124.57 \pm 45.98$ & NS & $70.71 \pm 37.88$ & $6.57 \pm 3.73$ & S \\
\hline \multicolumn{6}{|l}{ Confidence interval was evaluated at 95\%; significance p < 0.05; S=Significant; NS-not significant } \\
\hline
\end{tabular}

Table 4: Comparison of the effects of two brushing techniques on plaque scores

\begin{tabular}{|c|c|c|c|c|c|c|c|}
\hline & & PRE-3 & PRE-5 & sig. 2 TAILED & POST-3 & POST-5 & sig. 2 TAILED \\
\hline \multirow{2}{*}{$\begin{array}{l}\text { B R U S H - } \\
\text { ING TECH- } \\
\text { NIQUE }\end{array}$} & MBT & $185.25 \pm 33.83$ & $143.25 \pm 43.81$ & S & $40.35 \pm 26.41$ & $17.05 \pm 11.63$ & S \\
\hline & MST & $183.80 \pm 39.93$ & $168.15 \pm 41.13$ & S & $45.85 \pm 21.28$ & $9.65 \pm 6.20$ & S \\
\hline \multicolumn{8}{|c|}{ MBT: modified Bass technique; MST: modified Stillman's technique } \\
\hline \multicolumn{8}{|c|}{ Confidence interval was evaluated at $95 \%$; significance $\mathrm{p}<0.05 ; \mathrm{S}=$ Significant } \\
\hline
\end{tabular}

Table 5: Comparison of the combined effects of brushing techniques and grips on plaque removal

\begin{tabular}{|l|l|l|l|l|l|l|l|}
\hline & MBT & MST & & & & & \\
\hline & PRE-3 & PRE-5 & sig. 2 TAILED & PRE-3 & PRE-5 & sig. 2 TAILED & \\
\hline GRIP & $\begin{array}{l}\text { D I S T A L } \\
\text { OBLIQUE }\end{array}$ & $183.11 \pm 37.83$ & $138.22 \pm 50.87$ & S & $185.69 \pm 40.60$ & $174.00 \pm 42.98$ & NS \\
\hline & OBLIQUE & $192.62 \pm 23.12$ & $165.38 \pm 12.17$ & S & $184.00 \pm 37.32$ & $175.67 \pm 34.53$ & NS \\
\hline
\end{tabular}

\section{Discussion}

It is rational to assume that the efficiency of the brushing technique used is a major determinant of the effectiveness of plaque removal from the tooth surface. A great deal of research has been undertaken to investigate the relative effectiveness of different grips in this respect. Understanding the relationship between brushing technique and grip method, and the effects of each on plaque reduction, has major implications for clinicians.

The current study was conducted across five visits. In the first visit, researchers instructed subjects to brush their teeth in their usual manner. In normal circumstances, people brush their teeth with vigorous horizontal, circular, and/ or vertical movements. During this visit, as indicated in our analysis, the majority of subjects employed what is known as the horizontal scrubbing method. A number of studies have reported that horizontal scrubbing is the brushing technique of choice in younger subjects.

The horizontal scrubbing method is effective for the removal of plaque from the inner surfaces and smooth outer surfaces of teeth. Nonetheless, it is usually regarded as harmful. This is because vigorous horizontal scrubbing of teeth can promote gingival recession, and when combined with a sufficiently abrasive paste and a hard-textured toothbrush, it can expose some parts of the teeth to abrasion.
Subjects in the current study were shown how to brush their teeth using either the modified Bass technique or the modified Stillman's technique. Both of these techniques have the capacity to reduce gingival trauma and enhance the efficiency of biofilm removal from the tooth surface.

The current study also verified that the distal oblique grip is the most commonly used grip, as has been previously reported in other studies, such as those of Beals et al.,[7] Das et al,[3] and Mentes et al [12]. However, in the current study, we did not observe any subjects using either the spoon grip or the power grip.

When plaque scores from the first visit were compared for subjects using different grips, we noted some differences in the plaque scores but none were statistically significant. We did observe statistically significant differences between the plaque scores recorded on Visits 1 and 5 when comparing scores from subjects using the three different grip methods. The fact that the pre-brushing plaque scores did not differ significantly but the post-brushing plaque scores did suggests that the grip method used by subjects plays a critical role in plaque removal only when it is used in conjunction with an appropriate brushing technique. 
On the second visit, we divided the subjects into two groups comprised of 20 individuals per group. We taught one group how to brush using the modified Stillman's technique, and the other how to brush using the modified Bass technique. In both groups, the plaque scores recorded during the fifth visit were significantly better than those recorded during the third visit. From these findings, we can draw the conclusion that both brushing techniques are effective in improving plaque removal.

The findings of our study was contradictory to those of Sharma et al., [8] who reported that the modified Stillman's technique was efficacious with respect to plaque removal when tested in 100 school-age children. The results reported by Poyato-Ferrera et al. [4] concur with those of the current study, in that the modified Bass technique was also an efficacious method for removing plaque. Indeed, Poyoto-Ferrera et al. [4] reported that the modified Bass technique was the optimal method for brushing teeth in terms of plaque removal, and the current study was similar in that this method again yielded the most significant differences in plaque scores after brushing. The results of the current study contrast with the those of Aria et al, [9] who reported that the modified Bass technique was less effective for plaque removal than the modified Stillman's technique; however, our findings are in agreement with those of Saghazadeh et al. [13] who reported that, when plaque removal was compared between six different brushing techniques, the modified Bass technique was more effective than the modified Stillman's technique.

Lastly, the current study observed statistically significant differences between the pre-brushing plaque scores recorded at the third visit and those recorded at the fourth visit, when the modified Bass technique was being used in conjunction with either an oblique or a distal oblique grip. The results of the current study suggest that the modified Bass technique can be used effectively in conjunction with either an oblique distal grip or an oblique grip for plaque removal.

Our current research findings require validation by further clinical studies before it can be definitively concluded that the modified Bass technique offers superior plaque removal to other methods. Notably, a few issues may limit the generalization of the findings of the current study. First of all, due to the involvement of video recording in the research design some subjects may have felt intimidated and altered their toothbrush grip. Secondly, the sample size and composition may also have compromised the validity of the data, given that we only observed three types of toothbrush grips among our study subjects, who were all male and of similar age.

Nonetheless, this study has clinical implications because advice on maintaining oral hygiene is a key function of dental professionals, who can now recommend the combination of the modified Bass technique with a distal oblique or oblique grip for plaque reduction.

\section{Conclusion}

Despite the fact that a good grip affords greater dexterity when brushing the teeth, a good grip alone is not adequate for maintaining optimal oral health. Combining an appropriate technique for tooth brushing with an appropriate toothbrush grip is the most efficient way to reduce plaque on the teeth.

Our study confirms what other clinical studies have established: that the modified Bass technique is an effective method for plaque removal. It shows that the modified Bass technique is significantly more effective than the modified Stillman's technique. Furthermore, we have established that the use of the modified Bass technique in conjunction with a distal oblique grip or an oblique grip is more efficient than its use in conjunction with other grips. 


\section{References}

1) Robinson E. A (1976) comparative evaluation of the Scrub and Bass Methods of toothbrushing with flossing as an adjunct (in fifth and sixth graders) Am J Public Health 66: 1078-1081.

2) Sälzer S, Slot DE, Van der Weijden FA, Dörfer CE (2015) Efficacy of inter-dental mechanical plaque control in managing gingivitis--a meta-review. J ClinPeriodontol 42 Suppl 16:S92-105.

3) Das UM, Singhal P (2009) Tooth brushing skills for the children aged 3-11 years. J Indian Soc Pedod Prev Dent 27: 104-107.

4) Poyato-Ferrera M, Segura-Egea JJ, Bullón-Fernández P (2003) Comparison of modified Bass technique with normal toothbrushing practices for efficacy in supragingival plaque removal. Int J Dent Hyg 1: $110-114$.

5) Kimmelman BB, Tassman GC (1960) Research in designs of children's toothbrushes. J Dent Child 27: 60-64.

6) Mathur R, Jain S, Meena S, Parvez M (2013) A Comparative Evaluation of Commercially Available Pediatric Toothbrushes. Journal of Oral Health Research 2:13-17.

7) Beals D, Wong-Paredes M, Allen B, Rutter B, Stegemen J (1999) Grip architecture in manual toothbrushing. J Dent Res. 78: Spec, 413 (abstract).

8) Sharma S, Yeluri R, Jain AA, Munshi AK (2012) Effect of toothbrush grip on plaque removal during manual toothbrushing in children. J Oral Sci. 54: 183-190.

9) Arai T, Kinoshita S (1977) A comparison of plaque removal by different toothbrushes and tooth brushing methods. Bull Tokyo Med Dent Univ 24: 177-188.

10) Gill P, Stewart K, Treasure E, Chadwick B (2008) Methods of data collection in qualitative research: interviews and focus groups. $\mathrm{Br}$ Dent J 204: 291-295.

11) Turesky S, Gilmore ND, Glickman I (1970) Reduced plaque formation by the chloromethyl analogue of victamine C. J Clin Periodontol 41: 41-43.

12) Mentes A, Atukeren J (2002) A study of manual toothbrushing skills in children aged 3 to 11 years. J Clin Pediatr Dent 27: 91-94.

13) Saghazadeh M, Ashayeri N (2004)The comparison between the effectiveness of six different methods on removing dental bacterial plaque. Jdm 17: 26-38.

\footnotetext{
Submit your manuscript to a JScholar journal and benefit from:

ฯ Convenient online submission

ฯ Rigorous peer review

- Immediate publication on acceptance

q Open access: articles freely available online

9 High visibility within the field

ฯ Better discount for your subsequent articles

Submit your manuscript at

http://www.jscholaronline.org/submit-manuscript.php
} 